\title{
Development and validation of a risk score model for prediction of lower extremity arterial disease in Chinese with type 2 diabetes aged over 50 years
}

\author{
Xiaomei Zhang ${ }^{1}$, Zhangrong $\mathrm{Xu}^{2}$, Xingwu Ran ${ }^{3}$ and Linong $\mathrm{Ji}^{1,4}$ \\ ${ }^{1}$ Department of Endocrinology, Peking University International Hospital, Beijing, China \\ ${ }^{2}$ Diabetes Center, Characteristic Medical Center of Strategic Support Force, Beijing, China \\ ${ }^{3}$ Department of Endocrinology and Metabolism, West China Hospital, Sichuan University, Chengdu, China \\ ${ }^{4}$ Department of Endocrinology and Metabolism, Peking University People's Hospital, Beijing, China
}

Correspondence should be addressed to X Ran or L Ji: ranxingwu@163.com or jilinong@pkuih.edu.cn

\begin{abstract}
Background: Lower extremity arterial disease (LEAD) is highly prevalent in people with diabetes in China, but half of cases are underdiagnosed due to diversities of clinical presentations and complexities of diagnosis approaches. The purpose of this study was to develop a risk score model for LEAD to facilitate early screening among type 2 diabetes (T2DM) patients.

Methods: A total of 8313 participants with T2DM from the China DIA-LEAD study, a multicenter, cross-sectional epidemiological study, were selected as the training dataset to develop a risk score model for LEAD by logistic regression. The area under receiver operating characteristic curve (AUC) and bootstrapping were utilized for internal validation. A dataset of 287 participants consecutively enrolled from a teaching hospital between July 2017 and November 2017 was used as external validation for the risk score model.

Results: A total of 931 (11.2\%) participants were diagnosed as LEAD in the training dataset. Factors including age, current smoking, duration of diabetes, blood pressure control, low density lipoprotein cholesterol, estimated glomerular filtration rate, and coexistence of cardio and/or cerebrovascular disease correlated with LEAD in logistic regression analysis and resulted in a weighed risk score model of $0-13$. A score of $\geq 5$ was found to be the optimal cut-off for discriminating moderate-high risk participants with AUC of 0.786 (95\% Cl: 0.778-0.795). The bootstrapping validation showed that the AUC was 0.784 . Similar performance of the risk score model was observed in the validation dataset with AUC of 0.731 (95\% Cl: $0.651-0.811$ ). The prevalence of LEAD was $3.4,12.1$, and $27.6 \%$ in the low risk (total score $0-4$ ), moderate risk (total score 5-8), and high risk (total score 9-13) groups of LEAD in the training dataset, respectively, which were $4.3,19.6$, and $30.2 \%$ in the validation dataset.

Conclusion: The weighed risk score model for LEAD could reliably discriminate the presence of LEAD in Chinese with T2DM aged over 50 years, which may be helpful for a precise risk assessment and early diagnosis of LEAD.
\end{abstract}

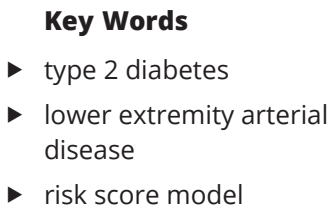

Endocrine Connections (2021) 10, 1212-1220

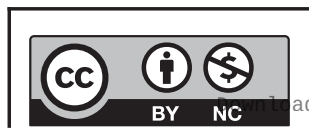

This work is licensed under a Creative Commons Attribution-NonCommercial 4.0 International License. ded from Bioscientifica.com at 04/26/2023 12:16:10PM 


\section{Introduction}

One of the major manifestations of systemic atherosclerosis in people with type 2 diabetes (T2DM) is the lower extremity arterial disease (LEAD), which contributes to an excess risk of major cardiovascular events and is commonly associated with a higher risk of both cardiovascular and all-cause mortality $(1,2,3)$. It also leads to a high rate of lower-limb adverse events and nontraumatic amputations $(4,5)$. The prevalence of LEAD among diabetic patients was very high and increased alarmingly in China, affecting over 26 million people among adult patients with diabetes (6).

Despite its burden on public health, clinicians and patients have a low level of awareness of LEAD. There is a lack of diagnosis in many patients, and those who are diagnosed as LEAD are undertreated with poor management of risk factors, even in western countries with an advanced healthcare system $(7,8)$. The China DIA-LEAD study reported that more than half of LEAD patients remained underdiagnosed and only less than one-third patients were treated (6). People with LEAD present a spectrum of symptoms from none to severe. Asymptomatic LEAD represents over $50 \%$ of patients with LEAD (7) and benefits most from risk factor management and regular follow-up. Timely screening and detection of LEAD is essential to avoid low extremity amputation or diabetic foot and reduce the cost of health care. Therefore, there is a need to develop a reliable and easy screening model for determining the presence of LEAD early for clinical practitioners, especially for the general practitioner in the primary care setting.

Epidemiologic evidence has demonstrated that the risk factors for LEAD vary greatly by race and geographic discrepancies $(9,10)$. Recently, using data from six U.S. community-based cohorts, Matsushita et al. developed risk calculators for the lifetime risk of LEAD and prevalent LEAD and suggested a higher lifetime risk of LEAD in Blacks compared with Whites and Hispanics (11). Most previous studies of LEAD risk scores have been in the general population $(11,12)$. A few studies conducted in T2DM population developed the risk scores mainly for low extremity amputation (13) or diabetic foot (14). This study aimed to develop and validate a risk score model for LEAD in Chinese with T2DM aged over 50 years, using data from a welldesigned epidemiologic study as the training source and data from the electronic medical records of a teaching hospital in Beijing as the validation dataset.

\section{Methods}

\section{Data source}

The China DIA-LEAD study, which has been described in detail previously, is a cross-sectional study representing different geographic regions of Mainland China and aimed to determine the epidemiological characteristics of LEAD in Chinese with T2DM (6). Participants with the following conditions were enrolled: (1) aged 50 years or older with a diagnosis of T2DM and at least one of the following risk factors: history of cardiovascular disease or cerebrovascular disease, hypertension, dyslipidemia, current smoking, and diabetic duration greater than 5 years; or (2) aged over 65 years with a diagnosis of T2DM. Patients with type 1 diabetes or gestational diabetes were excluded from this study. In total, 7113 inpatients and 3568 outpatients with T2DM were consecutively enrolled from 30 hospitals between June 2016 and January 2017. We excluded 2368 participants due to missing data of smoking, weight, height, blood pressure, LDL-C, or serum creatinine in this study, leaving 8313 participants as the training source for analysis. Two hundred and eighty-seven patients with T2DM were prospectively recruited during July 2017 and November 2017 and selected as the validation source in our analysis based on the same inclusion and exclusion criteria as the China DIA-LEAD study. The study protocol and informed consent document were reviewed and approved by the ethics committee of the Peking University International Hospital.

\section{Measurement and calculation of ankle-brachial index in diagnosing LEAD}

Ankle-brachial index (ABI) was measured with participants in the supine position. Trained investigators measured resting ankle and brachial systolic blood pressures at each site under a standardized protocol and equipment (Omron VP-1000, Omron Inc., China). The ABI results were available on the printed reports automatically. People with ABI equal or less than 0.9 were defined as having LEAD, according to local clinical practice and guideline recommendation (15).

\section{Data collection}

Participants' information was input into a welldesigned electronic medical record (reaching HIMSS stage 7) system (Beijing Healthvision Co., Ltd).
This work is licensed under a Creative Commons Attribution-NonCommercial 4.0 International License. ded from Bioscientifica.com at 04/26/2023 12:16:10PM 
Data regarding demographic characteristics, health behaviors, medical history, and medications were obtained by self-reporting. Alcohol consumption was defined as drinking $\geq 500 \mathrm{~mL}$ beer per day or daily red wine consumption of $\geq 150 \mathrm{~mL}$. A non-smoker was defined as someone who had not smoked one or more cigarettes during the past year before enrollment. Anthropometric measurements were conducted by trained nurses, adhering to standardized techniques. Height was measured using a stadiometer, and weight was measured using an electronic scale. BMI wascalculated by dividing the weightin kilograms by the square of the height in metres. Blood pressure was measured twice with an electronic sphygmomanometer in the sitting position (Omron HBP-9021, Omron Inc., China). The mean of the readings was recorded. Venous blood samples were taken, and local hospital laboratories were used for hemoglobin $\mathrm{A} 1 \mathrm{c}\left(\mathrm{HbA}_{1 \mathrm{c}}\right)$, serum creatinine, and lipid profile testing. The equation of the Chronic Kidney Disease Epidemiology Collaboration was used to calculate the estimated glomerular filtration rate (eGFR) (16).

\section{Statistical analysis}

SPSS 23.0 (SPSS Inc.) and SAS version 9.4 (SAS Institute Inc., Cary, NC) were used to conduct the statistical analysis. Those 8313 eligible participants from China DIA-LEAD study were selected as the training dataset for developing a weighed risk score model for LEAD in Chinese with T2DM, while 287 patients from Peking University International Hospital were used for validation of the developed risk score model. Data were presented as mean \pm S.D., median (Q1, Q3), or proportions. Differences were investigated by $t$-test if continuous variables or $\chi^{2}$ test if proportion variables as appropriate. The threshold for statistical significance was defined as $P<0.05$.

First, we identified factors individually associated with LEAD in participants with T2DM by univariate analysis with $P<0.10$, using the training dataset. Those factors demonstrating an individual association and factors clinically considered to be related to LEAD even without significance were carried forward in a stepwise multivariate logistic regression with criteria of 0.05. Continuous variables were dichotomized according to the cut-off values of optimal diagnostic performance (largest Youden index) determined by the receiver operating characteristic (ROC) curve or standard thresholds of clinical diagnosis or test. Therefore, age was categorized into $\geq 65$ years and $<65$ years. Duration of diabetes was categorized into $\geq 10$ years and $<10$ years. BMI was categorized into $<24,24-28$, and $\geq 28 \mathrm{~kg} / \mathrm{m}^{2} . \mathrm{HbA}_{1 \mathrm{c}}$ was categorized into $<7,7-9$, and $\geq 9 \%$. LDL-C was categorized into $\geq 2.6$ and $<2.6 \mathrm{mmol} / \mathrm{L}$. eGFR was categorized into $\geq 90 \mathrm{~mL} / \mathrm{min} / 1.73 \mathrm{~m}^{2}, 60-90 \mathrm{~mL} / \mathrm{min} /$ $1.73 \mathrm{~m}^{2}$, and $<60 \mathrm{~mL} / \mathrm{min} / 1.73 \mathrm{~m}^{2}$. Uncontrolled blood pressure was defined as either systolic blood pressure (SBP) $\geq 140 \mathrm{mmHg}$ or diastolic blood pressure (DBP) $\geq 90 \mathrm{mmHg}$. Variables included in the regression were then assigned the weighed score according to the $\beta$ coefficient of each variable divided by the lowest $\beta$ coefficient of the variable remained in the regression model, and the resulting quotient was rounded to the nearest whole number. Each participant's score was calculated both in the training dataset and the validation dataset.

Secondly, the discrimination and calibration of this model were evaluated by ROC curve with the area under the curve (AUC) and Hosmer-Lemeshow (H-L) goodnessof-fit test, respectively. The diagnostic performance of the risk score model was expressed as sensitivity, specificity, Youden index, positive predictive value (PPV), and negative predictive value (NPV). The bootstrap sampling approach was used to create new data to perform internal validation with 1000 bootstrap samples. The diagnostic abilities of the risk score model in the training dataset and validation dataset were compared using $\mathrm{Z}$ test. A $P$ value of less than 0.05 was considered statistically significant.

\section{Results}

\section{Characteristics of participants in the training dataset and validation dataset}

A total of 8313 participants from China DIA-LEAD study were included in our analysis as the training dataset. The characteristics of the training dataset classified by LEAD are shown in Table 1. Participants in LEAD group had older mean age and mean age of diagnosis of diabetes, longer duration of diabetes, higher SBP, lower DBP, and eGFR as compared to non-LEAD group. The prevalence of smoking, alcohol consumption, and macro- and microvascular complications in LEAD group was significantly higher than that in non-LEAD group. More participants were taking antiplatelet drugs in LEAD group. Notably, there were disparities of participants' profiles between the training and validation datasets in terms of gender, smoking status, family history of premature cardiovascular disease (CVD), BMI, LDL-C, eGFR and some of diabetic complications (Table 2). However, age, duration of diabetes, and alcohol consumption were comparable in both datasets. A total of 931 (11.2\%) and 39 (13.6\%) participants were diagnosed as LEAD in the two datasets, respectively. 
Table 1 Demographic and clinical features of participants.

\begin{tabular}{l}
\hline Variables \\
\hline Gender, males, \% $(n)$ \\
Age, years \\
Duration of diabetes, years \\
Age at diagnosis of diabetes, years \\
Current smoking, \% ( $n)$ \\
Alcohol consumptions, \% ( $n)$ \\
Family history of premature CVD, \% ( $n)$ \\
BMI, kg/m² \\
SBP, mmHg \\
DBP, mmHg \\
HbA ${ }_{1 c} \%$ \\
TG, mmol/L \\
LDL-C, mmol/L \\
eGFR, mL/min/1.73 m² \\
Macrovascular complications, \% (n) \\
Cardiovascular disease, \% $(n)$ \\
Cerebrovascular disease, \% $(n)$ \\
Microvascular complications, \% ( $(n)$ \\
Diabetes kidney disease, \% $(n)$ \\
Diabetic retinopathy, \% $(n)$ \\
Distal symmetric polyneuropathy, \% (n) \\
Antiplatelet therapy, \% $(n)$ \\
Statins therapy, \% $(n)$
\end{tabular}

\begin{tabular}{c}
\hline Total $(n=8313)$ \\
\hline $54.2(4504)$ \\
$63.8 \pm 9.0$ \\
$10(5,15)$ \\
$53.5 \pm 9.8$ \\
$27.1(2256)$ \\
$15.6(1295)$ \\
$5.4(449)$ \\
$24.9 \pm 3.4$ \\
$135(123-148)$ \\
$80(70-86)$ \\
$8.5 \pm 2.1$ \\
$1.90 \pm 1.60$ \\
$2.72 \pm 0.96$ \\
$86.8 \pm 24.9$ \\
$27.5(2285)$ \\
$15.0(1246)$ \\
$17.0(1416)$ \\
$66.1(5495)$ \\
$20.0(1664)$ \\
$20.8(1729)$ \\
$47.2(3923)$ \\
$38.6(3205)$ \\
$45.5(3780)$
\end{tabular}

\section{Establishing a risk score model for LEAD in training dataset}

In the training dataset, a total of eight factors were associated with LEAD in univariate analysis with $P<0.10$, including males, older age, longer duration of diabetes, current smoking, uncontrolled blood pressure, lower eGFR, coexistence with cardio-and/or cerebrovascular disease, and coexistence with microvascular complications. Another two factors (alcohol consumption and LDL-C) that

\begin{tabular}{c}
\hline LEAD $(n=931)$ \\
\hline $49.7(463)$ \\
$69.3 \pm 9.5$ \\
$10(5,18)$ \\
$55.8 \pm 11.1$ \\
$30.9(288)$ \\
$15.7(1157)$ \\
$4.9(46)$ \\
$24.9 \pm 3.5$ \\
$140(126-156)$ \\
$78(70-86)$ \\
$8.6 \pm 2.0$ \\
$1.90 \pm 1.48$ \\
$2.79 \pm 1.01$ \\
$76.6 \pm 26.9$ \\
$48.1(448)$ \\
$26.2(244)$ \\
$33.6(313)$ \\
$79.6(741)$ \\
$30.6(285)$ \\
$31.8(296)$ \\
$62.2(579)$ \\
$46.6(434)$ \\
$47.5(442)$
\end{tabular}

\begin{tabular}{c}
\hline Non-LEAD $(n=7382)$ \\
\hline $54.7(4041)$ \\
$63.1 \pm 8.7$ \\
$8(4,14)$ \\
$53.3 \pm 9.6$ \\
$26.7(1968)$ \\
$14.8(1131)$ \\
$5.5(406)$ \\
$24.9 \pm 3.4$ \\
$134(122-147)$ \\
$80(71-86)$ \\
$8.5 \pm 2.1$ \\
$1.90 \pm 1.61$ \\
$2.71 \pm 0.95$ \\
$88.1 \pm 24.2$ \\
$24.9(1831)$ \\
$13.6(1002)$ \\
$14.9(1103)$ \\
$64.5(4758)$ \\
$18.7(1379)$ \\
$19.4(1434)$ \\
$45.3(3345)$ \\
$37.5(2771)$ \\
$45.2(3338)$
\end{tabular}

\begin{tabular}{rr}
\hline P value \\
\hline 0.004 \\
$\leq 0.001$ \\
$\leq 0.001$ \\
$\leq 0.001$ \\
0.006 \\
0.024 \\
0.539 \\
0.429 \\
$\leq 0.001$ \\
0.001 \\
0.311 \\
0.608 \\
0.087 \\
$\leq 0.00$ \\
$\leq 0.001$ \\
$\leq 0.001$ \\
$\leq 0.001$ \\
$\leq 0.001$ \\
$\leq 0.001$ \\
$\leq 0.001$ \\
$\leq 0.001$ \\
$\leq 0.001$ \\
0.196
\end{tabular}

were not statistically different but clinically critical to LEAD development were also carried into the multiple regression model. Of the candidate variables analyzed, seven factors (age, duration of diabetes, smoking status, blood pressure control, LDL-C, eGFR, and coexistence of cardioand/or cerebrovascular disease) remained significant in stepwise multivariate logistic regression, and weight scores were assigned to each risk factor consequently. A cumulated risk score ranging from 0 to 13 was generated based on $\beta$ coefficient in above-mentioned multivariate

Table 2 Participants' characteristics in the training dataset and validation dataset.

\begin{tabular}{l} 
Variables \\
\hline Gender, males, \% $(n)$ \\
Age, years \\
Duration of diabetes, years \\
Current smoking, \% $(n)$ \\
Alcohol consumptions, \% ( $n)$ \\
Family history of premature CVD, \% ( $n)$ \\
BMI, kg/m² \\
SBP, mmHg \\
DBP, mmHg \\
LDL-C, mmol/L \\
eGFR, mL/min/1.73 m² \\
Diabetic complications \\
Cardiovascular disease, \% $(n)$ \\
Cerebrovascular disease, \% $(n)$ \\
$\quad$ Diabetes kidney disease, \% $(n)$ \\
Diabetic retinopathy, \% $(n)$ \\
Distal symmetric polyneuropathy, \% $(n)$
\end{tabular}

\begin{tabular}{l} 
Valid \\
\hline NC \\
\hline
\end{tabular}

This work is licensed under a Creative Commons Attribution-NonCommercial 4.0 International License.
$15.0(1246)$

$17.0(1416)$

$20.0(1664)$

20.8 (1729)

$47.2(3923)$ alidation dataset $(n=289)$

$51.6(148)$

$62.7 \pm 8.8$

$10(4,15)$

$35.9(103)$

$18.2(52)$

$1.0(3)$

$26.1 \pm 4.1$

$130(120,140)$

$80(72,83)$

$2.62 \pm 0.84$

$95.0 \pm 18.8$

$15.3(44)$

$43.9(126)$

$22.3(64)$

$19.9(57)$

$21.3(61)$

\begin{tabular}{c}
\hline P value \\
\hline$<0.05$ \\
0.050 \\
0.501 \\
$<0.05$ \\
0.290 \\
$<0.05$ \\
$<0.05$ \\
$<0.05$ \\
0.257 \\
$<0.05$ \\
$<0.05$
\end{tabular}

0.950

$<0.05$

$<0.05$

0.760

$<0.05$ 
Table 3 Clinical predictors for LEAD identified by the multivariate logistic regression analysis.

\begin{tabular}{llc}
\hline Variables & & $\beta$ \\
\hline Age ( $\geq 65$ years vs $<65$ years) & & 0.892 \\
Current smoking (yes vs no) & & 0.429 \\
Duration of diabetes ( $\geq 10$ years vs $<10$ years) & & 0.529 \\
Cardio- and/or cerebrovascular disease (yes vs no) & 0.781 \\
Blood pressure control (no vs yes) & & 0.459 \\
LDL-C ( $\geq 2.6$ vs. $<2.6 \mathrm{mmol} / \mathrm{L})$ & & 0.313 \\
eGFR $\left(<60\right.$ vs $\left.90 \mathrm{~mL} / \mathrm{min} / 1.73 \mathrm{~m}^{2}\right)$ & 0.566 \\
\hline
\end{tabular}

logistic regression (Table 3). One score increase could lead to $33 \%$ increase risk of LEAD (OR 1.330, 95\% CI: 1.298-1.363).

Supplementary Tables 1 and 2 (see section on supplementary materials given at the end of this article) show the results of logistic regression analyses using continuous variables and including medication, respectively.

\section{Validation of the risk score model for LEAD}

The risk score model showed good discrimination (AUC $=0.786 ; 95 \%$ CI: $0.778-0.795, P \leq 0.001$ ) and calibration (Hosmer-Lemeshow test: $\chi^{2}=11.223, P>0.05$ ) in the training dataset and an optimal cut-off value of $\geq 5$ could predict the presence of LEAD with sensitivity of $79.5 \%$ and specificity of $66.0 \%$ (Youden index $=0.355$ ) (Fig. 1A). The risk score under 5 was defined as low risk, a score from 5 to 8 was defined as moderate risk, and a score over 8 was defined as high risk of LEAD. The prevalence of LEAD was $3.4,12.1$, and $27.6 \%$ in the low risk, moderate risk, and high risk groups of LEAD, respectively, in the training dataset.

The risk score was retrospectively calculated for each participant in the validation dataset. The prevalence of LEAD increased from $4.3 \%(n=6)$ at low-risk group to $19.6 \%(n=20)$ and overwhelmingly high to $30.2 \%(n=13)$ at moderate- and high-risk groups, respectively, in the validation dataset. The risk score model also demonstrated good discrimination (AUC $=0.738$; 95\% CI: 0.684-0.788, $P \leq 0.001$ ) (Fig. 1B) and calibration (Hosmer-Lemeshow test: $\left.\chi^{2}=7.360, P>0.05\right)$ in the validation dataset. AUC of the risk score model for distinguishing participants with LEAD in the training dataset showed no statistically significant difference with that in the validation dataset $(\mathrm{Z}=-1.278, P=0.899)$. Table 4 presents the sensitivity, specificity, PPV, and NPV for different risk score cut-off values ( 5 and 9 ) in the validation dataset to evaluate the predictive performance. Hence, we further developed an easy-going questionnaire for screening high-risk people with LEAD among T2DM in clinical practice (Table 5).

\begin{tabular}{c}
\hline OR $(95 \% \mathrm{Cl})$ value \\
\hline $2.441(2.081-2.862)$ \\
$1.536(1.312-1.798)$ \\
$1.697(1.458-1.975)$ \\
$2.184(1.889-2.525)$ \\
$1.583(1.371-1.827)$ \\
$1.368(1.183-1.580)$ \\
$1.761(1.450-2.139)$ \\
\hline
\end{tabular}

\begin{tabular}{l}
\hline P value \\
\hline$\leq 0.001$ \\
$\leq 0.001$ \\
$\leq 0.001$ \\
$\leq 0.001$ \\
$\leq 0.001$ \\
$\leq 0.001$ \\
$\leq 0.001$ \\
\hline
\end{tabular}

\begin{tabular}{c}
\hline Translated into score \\
\hline 3 \\
1 \\
2 \\
3 \\
1 \\
1 \\
2 \\
\hline
\end{tabular}

\section{Discussion}

In this study, we investigated the risk factors for LEAD among Chinese with T2DM aged over 50 years and developed a risk score model based on easily accessible demographic and clinical variables. Our model composed of seven variables including age, duration of diabetes, smoking status, blood pressure control, coexistence of cardio- and/or cerebrovascular disease, LDL-C, and eGFR. These can all be easily evaluated in daily practice, demanding up to a questionnaire and a simple blood test. With the cut-off point of 5 , the sensitivity and specificity of the risk score model for predicting the presence of LEAD were $79.5 \%$ and $66.0 \%$ in the training dataset. To test the utility of this risk score model, we undertook internal and external validation analyses, and the model also demonstrated good ability of discrimination and calibration. The application of this simple risk score model to the general T2DM population aged over 50 years may serve as a first step to identify high-risk individuals and help to improve the early detection and proper management of LEAD.

In the literature, numerous risk factors associated with LEAD among T2DM have been reported (17, 18, 19, 20, 21). Of the seven predictors in our study, age and duration of diabetes are two non-modifiable factors. Age is a great promoter of atherosclerosis. LEAD is usually discovered after 50 years of age. Recently, global estimates revealed that LEAD prevalence increased with age, from a relatively uncommon disorder in people younger than 40 years to a common problem affecting around $10 \%$ of people aged 70 years, and about $15-25 \%$ of people aged over 80 years (22). In this study, age $\geq 65$ years was associated with 2.4 fold higher risk of developing LEAD compared to the age of 50-65 years. Besides, it is not surprising that the duration of diabetes is included in this model with a $66 \%$ increase of the risk of LEAD in those with duration of diabetes $\geq 10$ years relative to $<10$ years. Diabetes is closely related to the development of atherosclerotic diseases, including LEAD. The UK Prospective Diabetes Study showed that 

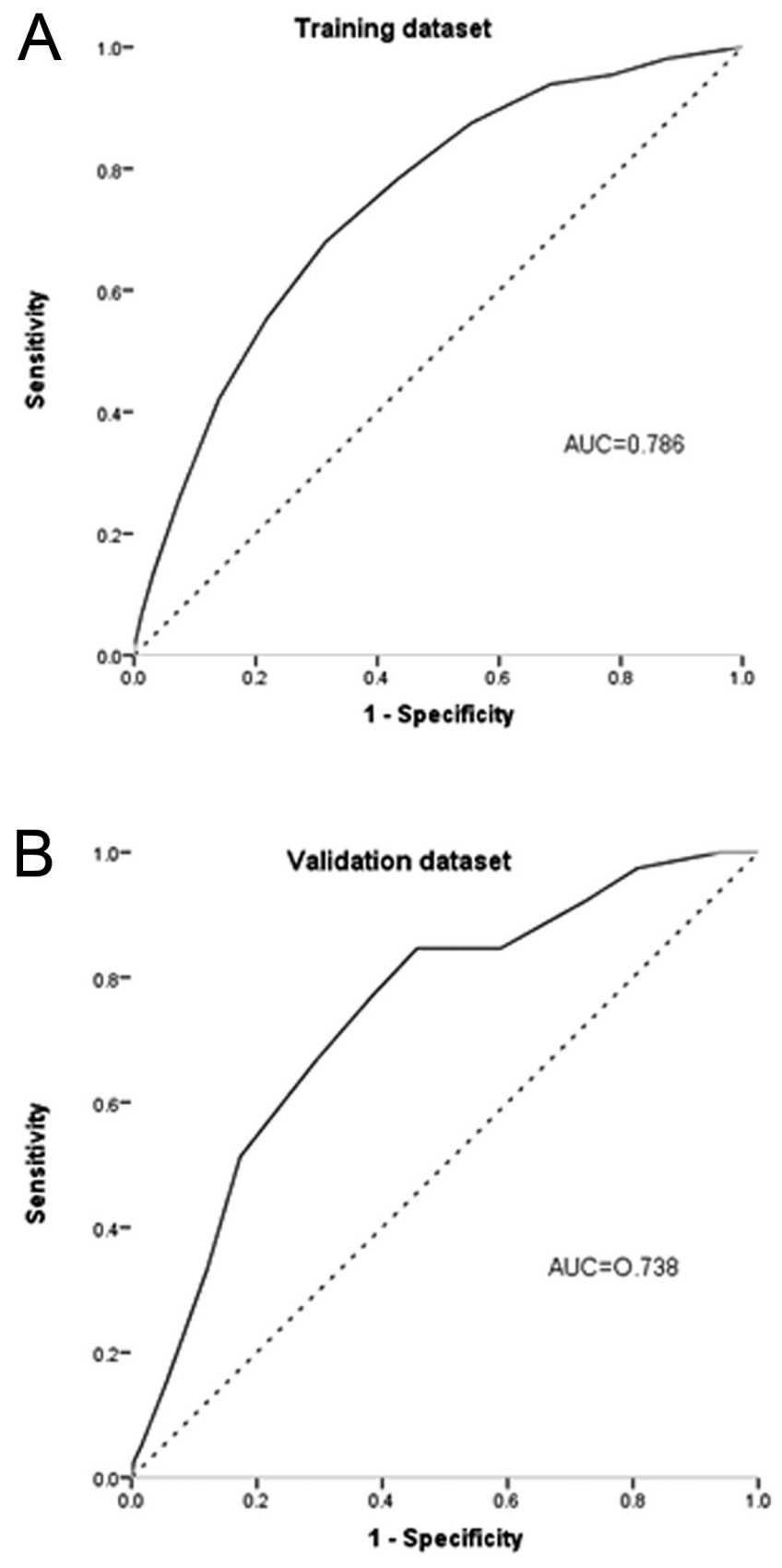

Figure 1

Diagnostic performance of the risk score model in the training dataset (A) and validation dataset (B).

the prevalence of LEAD was $1.2 \%$ at diagnosis of diabetes whereas it increased to $12.5 \%$ after 18 years of diabetes (18).

Modifiable risk factors included in our model are current smoking, blood pressure control, and LDL-C. Current smoking has been shown to increase the risk of LEAD by 70\% in comparison with nonsmoking in our study. This is consistent with most studies with some estimates of a two- to four-fold higher risk of LEAD in current smoking
Table 4 Performance of the score model at different cut-off points in the validation dataset.

\begin{tabular}{|c|c|c|}
\hline & \multicolumn{2}{|c|}{ Cut-off points } \\
\hline & $\geq 5$ points & $\geq 9$ points \\
\hline Sensitivity (95\% CI) & $0.846(0.695-0.941)$ & $0.333(0.191-0.502)$ \\
\hline Specificity $(95 \% \mathrm{CI})$ & $0.544(0.48-0.607)$ & $0.879(0.832-0.917)$ \\
\hline $\begin{array}{l}\text { Positive predictive } \\
\text { value }(95 \% \mathrm{Cl})\end{array}$ & $0.226(0.161-0.302)$ & $0.302(0.171-0.461)$ \\
\hline $\begin{array}{l}\text { Negative predictive } \\
\text { value }(95 \% \mathrm{Cl})\end{array}$ & 0.957 (0.909-0.984) & $0.893(0.848-0.929)$ \\
\hline
\end{tabular}

$(10,22,23,24)$. Some studies also demonstrated a doseresponse relationship between smoking and $\operatorname{LEAD}(23,25)$ and a reduced risk of LEAD after cessation of smoking (23), which could not be explored in our study. Hypertension and dyslipidemia are another two well-established risk factors for LEAD. Hypertension has been shown to be associated with 1.5- to 2-fold higher risk of LEAD in most epidemiological studies $(9,23,26)$. When examining blood pressure levels, higher SBP has been usually found to correlate with $\operatorname{LEAD}(3,10,27)$, whereas the association between DBP and LEAD is controversial $(3,18,26)$. Our study found a positive association of SBP with LEAD and a negative association of DBP with LEAD. Due to the inconsistent results between DBP and LEAD reported in the literature, we used blood pressure control for further multivariable regression analysis to establish the risk score model. We found that uncontrolled blood pressure increased 50\% risk of LEAD in comparison with controlled blood pressure. Dyslipidemia has been proved to relate to an increased risk of LEAD. Among lipid measurements, total cholesterol (TCHO), triglyceride (TG), LDL-C, HDL-C, and TCHO/HDL-C ratio have been reported as predictors for the development of LEAD in various studies $(19,23,24$, 26), which tends to attribute to collective effects of lipids. We observed that LDL-C was positively associated with the presence of LEAD, and LDL-C $\geq 2.6 \mathrm{mmol} /$ Lincreased nearly $40 \%$ risk of LEAD as compared to LDL-C $<2.6 \mathrm{mmol} / \mathrm{L}$. These findings have critical public health implications for healthcare providers and policy makers to develop effective strategies for the prevention and intervention of LEAD.

LEAD has been increasingly recognized as an associated condition in the context of diseases such as cardio or cerebrovascular disease and chronic kidney disease. As atherosclerosis is a systemic process, there exists a strong correlation between cardio- and/or cerebrovascular disease and LEAD due to shared common risk factors. Our study suggested that coexistence of cardio- and/or cerebrovascular disease was strongly associated with the risk of LEAD among T2DM. The relationship between chronic kidney disease and atherosclerosis disease 
Table 5 LEAD risk scores for Chinese with type 2 diabetes aged over 50 years.

\begin{tabular}{|c|c|c|}
\hline Variables & Categories & Score \\
\hline Age & $\begin{array}{l}\geq 65 \text { years } \\
<65 \text { years }\end{array}$ & $\begin{array}{l}3 \\
0\end{array}$ \\
\hline Current smoking status & $\begin{array}{l}\text { Yes } \\
\text { No }\end{array}$ & $\begin{array}{l}1 \\
0\end{array}$ \\
\hline Duration of diabetes & $\begin{array}{l}\geq 10 \text { years } \\
<10 \text { years }\end{array}$ & $\begin{array}{l}2 \\
0\end{array}$ \\
\hline Coexistence of cardio and/or cerebravascular disease & $\begin{array}{l}\text { Yes } \\
\text { No }\end{array}$ & $\begin{array}{l}3 \\
0\end{array}$ \\
\hline Blood pressure control & $\begin{array}{l}\text { Yes } \\
\text { No }\end{array}$ & $\begin{array}{l}1 \\
0\end{array}$ \\
\hline LDL-C & $\begin{array}{l}\geq 2.6 \mathrm{mmol} / \mathrm{L} \\
<2.6 \mathrm{mmol} / \mathrm{L}\end{array}$ & $\begin{array}{l}1 \\
0\end{array}$ \\
\hline eGFR & $\begin{array}{l}<60 \mathrm{~mL} / \mathrm{min} / 1.73 \mathrm{~m}^{2} \\
\geq 60 \mathrm{~mL} / \mathrm{min} / 1.73 \mathrm{~m}^{2}\end{array}$ & $\begin{array}{l}2 \\
0\end{array}$ \\
\hline
\end{tabular}

Risk for the presence of LEAD: low risk (total score 0-4); moderate risk (total score 0-4); high risk (total score 9-13).

including LEAD has been examined both in cross-sectional and prospective studies $(28,29,30)$. In a recent large meta-analysis of 21 prospective cohort studies including over 800,000 individuals without LEAD, Matsushita et al. found that lower eGFR was related to a 1.2-2 times higher incidence of LEAD depending on the severity of renal disease (29). The addition of eGFR significantly improved LEAD risk discrimination beyond traditional risk factors (10). Our findings corroborate the results reporting 1.7 times higher risk of LEAD among people with T2DM and eGFR $<60 \mathrm{~mL} / \mathrm{min} / 1.73 \mathrm{~m}^{2}$. Therefore, among people with conditions like cardio and/or cerebrovascular disease or chronic kidney disease, LEAD may be underestimated and should be actively screened for.

A few groups have proposed models to identify intermittent claudication $(12,31)$, which accounts for only $10-20 \%$ of LEAD. Many people with LEAD report no symptoms, even with moderately severe disease (7). Tools that predict LEAD are fewer. In addition, evidence suggests that for CVD prediction, diabetes-specific models may perform better than models from the general population $(32,33)$. However, studies involving the prediction of LEAD among T2DM are far fewer. Previously, our group has developed a nomogram to predict the occurrence of LEAD in people with $\mathrm{T} 2 \mathrm{DM}$, including age, gender, duration of diabetes, smoking, $\mathrm{HbA}_{1 \mathrm{c}}$, coexistence of coronary heart disease, and coexistence of diabetic microvascular complications (34). Although the developed nomogram is a simple-to-use and intuitive method to predict the risk of LEAD, sometimes indices such as microvascular complications are not available due to the lack of screening or monitoring in real clinical practice, which might hinder the wide use of this tool, especially at the primary care level.
A national survey in China has revealed that only onethird of patients with diabetes had regular examination of the eyes (35). The risk score model developed in this study includes simple clinical variables that can be obtained from a typical clinic visit.

Based on nationally representative data, our findings provide an easy and effective approach to estimate risk for the presence of LEAD among middle- and old-aged people with T2DM. However, some limitations of our study should be addressed. First, our risk score model was derived and validated both in cross-sectional data; therefore, the temporal relationship between risk factors and LEAD cannot be established, and the model might be unable to precisely predict the risk of incident LEAD in the future. Second, potentially important risk factors, such as glycemic variability or ethnicity were not investigated in our study owing to the lack of data. Studies have demonstrated that greater glycemic fluctuation may promote the occurrence and development of LEAD through aggravating vascular endothelial injury among diabetes (36). However, the evaluation of glycemic fluctuation requires a continuous glucose monitoring system or mathematical calculations based on more than two glycemic measurements, which cannot be easily acquired by primary practitioners. The risk of LEAD may also vary according to differences in ethnicity. Matsushita et al. reported higher risk of LEAD in Blacks than in Whites (11). The incidence of major LEAD was lower in Asians compared with those from Eastern Europe in ADVANCE study (37). Although China is a multi-ethnic country, Han nationality is the main nationality, accounting for more than $90 \%$ of the total population. Additional studies are warranted to assess this issue in China. We did not include novel risk factors,
This work is licensed under a Creative Commons Attribution-NonCommercial 4.0 International License. ded from Bioscientifica.com at 04/26/2023 12:16:10PM 
such as chronic inflammation biomarkers, homocysteine, or genotypes, which are not routinely measured. Third, smoking and alcohol use were only defined by current status, and the data were not available on quantitative measurements of smoking exposure and alcohol consumption, so dose-response relationship with LEAD cannot be explored. Fourth, an ABI $\leq 0.9$ was used to diagnose LEAD in our study. However, an ABI value of more than 1.3 is also considered as abnormal in some cases, reflecting calcified and stiffed arteries. Thus, the utility of $\mathrm{ABI} \leq 0.9$ to diagnose LEAD may lead to underestimating the true burden. Finally, our analysis was restricted to the $77.8 \%$ of the study population because of unavailability of data, which may result in selection bias. In order to assess the possibility of selection bias, we compared the characteristics of participants included in the study and those with missing data and excluded from the analysis (Supplementary Table 3). We found no significant difference in age, age at diagnosis of diabetes, BMI and $\mathrm{HbA}_{1 \mathrm{c}}$, and similar distributions in diabetic complications and medication use, which demonstrated the selection error might be random, resulting in the effect toward the null. Additionally, our population was derived from a national survey involving only people with T2DM aged over 50 years, so the findings may not be generalized to younger age groups.

\section{Conclusions}

Our study has presented a risk score model for predicting the risk of occurrence of LEAD among middle- and oldaged people with T2DM based on a national representative sample, which can be easily used in communities and clinical settings. Future prospective research is needed to verify the usefulness and feasibility of this model and identify ways to improve the accuracy of it in clinical practice.

\section{Supplementary materials}

This is linked to the online version of the paper at https://doi.org/10.1530/ EC-21-0152.

\section{Declaration of interest}

The authors declare that there is no conflict of interest that could be perceived as prejudicing the impartiality of the research reported.

\section{Funding}

This study was supported by an unrestricted research grant offered by Beijing Tide Pharmaceutical Co., LTD. and Bethune Charitable Foundation.

\section{Acknowledgements}

The authors would like to thank all China DIA-LEAD study investigators for their contribution to the successful completion of this study.

\section{References}

1 Shah B, Rockman CB, Guo Y, Chesner J, Schwartzbard AZ, Weintraub HS, Adelman MA, Riles TS \& Berger JS. Diabetes and vascular disease in different arterial territories. Diabetes Care 201437 1636-1642. (https://doi.org/10.2337/dc13-2432)

2 Criqui MH, Langer RD, Fronek A, Feigelson HS, Klauber MR, McCann TJ \& Browner D. Mortality over a period of 10 years in patients with peripheral arterial disease. New England Journal of Medicine 1992326 381-386. (https://doi.org/10.1056/ NEJM199202063260605)

3 Norman PE, Davis WA, Bruce DG \& Davis TM. Peripheral arterial disease and risk of cardiac death in type 2 diabetes: the Fremantle Diabetes Study. Diabetes Care 200629 575-580. (https://doi. org/10.2337/diacare.29.03.06.dc05-1567)

4 Agarwal S, Sud K \& Shishehbor MH. Nationwide trends of hospital admission and outcomes among critical limb ischemia patients: from 2003 to 2011. Journal of the American College of Cardiology 201667 1901-1913. (https://doi.org/10.1016/j.jacc.2016.02.040)

5 Boulton AJ, Vileikyte L, Ragnarson-Tennvall G \& Apelqvist J. The global burden of diabetic foot disease. Lancet 2005366 1719-1724. (https://doi.org/10.1016/S0140-6736(05)67698-2)

6 Zhang X, Ran X, Xu Z, Cheng Z, Shen F, Yu Y, Gao L, Chai S, Wang C, Liu J, et al. Epidemiological characteristics of lower extremity arterial disease in Chinese diabetes patients at high risk: a prospective, multicenter, cross-sectional study. Journal of Diabetes and Its Complications 201832 150-156. (https://doi.org/10.1016/j. jdiacomp.2017.10.003)

7 Hirsch AT, Criqui MH, Treat-Jacobson D, Regensteiner JG, Creager MA, Olin JW, Krook SH, Hunninghake DB, Comerota AJ, Walsh ME, et al. Peripheral arterial disease detection, awareness, and treatment in primary care. JAMA 2001286 1317-1324. (https://doi.org/10.1001/ jama.286.11.1317)

8 Bhatt DL, Steg PG, Ohman EM, Hirsch AT, Ikeda Y, Mas JL, Goto S, Liau CS, Richard AJ, Rother J, et al. International prevalence, recognition, and treatment of cardiovascular risk factors in outpatients with atherothrombosis. JAMA 2006295 180-189. (https:// doi.org/10.1001/jama.295.2.180)

9 Allison MA, Criqui MH, McClelland RL, Scott JM, McDermott MM, Liu K, Folsom AR, Bertoni AG, Sharrett AR, Homma S, et al. The effect of novel cardiovascular risk factors on the ethnic-specific odds for peripheral arterial disease in the Multi-Ethnic Study of Atherosclerosis (MESA). Journal of the American College of Cardiology 200648 1190-1197. (https://doi.org/10.1016/j.jacc.2006.05.049)

10 Criqui MH, Vargas V, Denenberg JO, Ho E, Allison M, Langer RD, Gamst A, Bundens WP \& Fronek A. Ethnicity and peripheral arterial disease: the San Diego Population Study. Circulation 2005112 2703-2707. (https://doi.org/10.1161/CIRCULATIONAHA.105.546507)

11 Matsushita K, Sang Y, Ning H, Ballew SH, Chow EK, Grams ME, Selvin E, Allison M, Criqui M, Coresh J, et al. Lifetime risk of lowerextremity peripheral artery disease defined by ankle-brachial index in the United States. Journal of the American Heart Association 20198 e012177. (https://doi.org/10.1161/JAHA.119.012177)

12 Leng GC \& Fowkes FG. The Edinburgh claudication questionnaire: an improved version of the WHO/Rose questionnaire for use in epidemiological surveys. Journal of Clinical Epidemiology 199245 1101-1109. (https://doi.org/10.1016/0895-4356(92)90150-1)

13 Li CI, Lin CC, Cheng HM, Liu CS, Lin CH, Lin WY, Wang MC, Yang SY $\&$ Li TC. Derivation and validation of a clinical prediction model for assessing the risk of lower extremity amputation in patients with type https://ec.bioscientifica.com

https://doi.org/10.1530/EC-21-0152 (c) 2021 The authors Published by Bioscientifica Ltc
This work is licensed under a Creative Commons Attribution-NonCommercial 4.0 International License. ded from Bioscientifica.com at 04/26/2023 12:16:10PM 
2 diabetes. Diabetes Research and Clinical Practice 2020165108231. (https://doi.org/10.1016/j.diabres.2020.108231)

14 Mills JL, Sr, Conte MS, Armstrong DG, Pomposelli FB, Schanzer A, Sidawy AN \& Andros G. The society for vascular surgery lower extremity threatened limb classification system: risk stratification based on wound, ischemia, and foot infection. Journal of Vascular Surgery 201459 e221-e222. (https://doi.org/10.1016/j. jvs.2013.08.003)

15 Society CD. Guidelines for screening and management of lower extremities arterial disease in type 2 diabetes. China Journal of Diabetes Mellitus 201305 82-88. (https://doi.org/10.3760/cma.j.i ssn.1674-5809.2013.02.006)

16 Levey AS, Stevens LA, Schmid CH, Zhang YL, Castro AF, 3rd, Feldman HI, Kusek JW, Eggers P, Van Lente F, Greene T, et al. A new equation to estimate glomerular filtration rate. Annals of Internal Medicine 2009150 604-612. (https://doi.org/10.7326/0003-4819-1509-200905050-00006)

17 Althouse AD, Abbott JD, Forker AD, Bertolet M, Barinas-Mitchell E, Thurston RC, Mulukutla S, Aboyans V, Brooks MM \& BARI 2D Study Group. Risk factors for incident peripheral arterial disease in type 2 diabetes: results from the bypass angioplasty revascularization investigation in type 2 diabetes (Bari 2D) trial. Diabetes Care 201437 1346-1352. (https://doi.org/10.2337/dc13-2303)

18 Adler AI, Stevens RJ, Neil A, Stratton IM, Boulton AJ \& Holman RR. UKPDS 59: hyperglycemia and other potentially modifiable risk factors for peripheral vascular disease in type 2 diabetes. Diabetes Care 200225 894-899. (https://doi.org/10.2337/diacare.25.5.894)

19 Selvin E \& Erlinger TP. Prevalence of and risk factors for peripheral arterial disease in the United States: results from the National Health and Nutrition Examination Survey, 1999-2000. Circulation 2004110 738-743. (https://doi.org/10.1161/01.CIR.0000137913.26087.F0)

20 Lu L, Mackay DF \& Pell JP. Meta-analysis of the association between cigarette smoking and peripheral arterial disease. Heart 2014100 414-423. (https://doi.org/10.1136/heartjnl-2013-304082)

21 Ridker PM, Stampfer MJ \& Rifai N. Novel risk factors for systemic atherosclerosis: a comparison of C-reactive protein, fibrinogen, homocysteine, lipoprotein(a), and standard cholesterol screening as predictors of peripheral arterial disease. JAMA 2001285 2481-2485. (https://doi.org/10.1001/jama.285.19.2481)

22 Fowkes FG, Rudan D, Rudan I, Aboyans V, Denenberg JO, McDermott MM, Norman PE, Sampson UK, Williams LJ, Mensah GA, et al. Comparison of global estimates of prevalence and risk factors for peripheral artery disease in 2000 and 2010: a systematic review and analysis. Lancet 2013382 1329-1340. (https://doi.org/10.1016/S01406736(13)61249-0)

23 Joosten MM, Pai JK, Bertoia ML, Rimm EB, Spiegelman D, Mittleman MA \& Mukamal KJ. Associations between conventional cardiovascular risk factors and risk of peripheral artery disease in men JAMA 2012308 1660-1667. (https://doi.org/10.1001/jama.2012.13415)

24 Wattanakit K, Folsom AR, Selvin E, Weatherley BD, Pankow JS, Brancati FL \& Hirsch AT. Risk factors for peripheral arterial disease incidence in persons with diabetes: the Atherosclerosis Risk in Communities (ARIC) study. Atherosclerosis 2005180 389-397. (https:// doi.org/10.1016/j.atherosclerosis.2004.11.024)

25 Merino J, Planas A, Elosua R, de Moner A, Gasol A, Contreras C, Vidal-Barraquer $\mathrm{F} \&$ Clara A. Incidence and risk factors of peripheral arterial occlusive disease in a prospective cohort of 700 adult elderly men followed for 5 years. World Journal of Surgery 201034 1975-1979. (https://doi.org/10.1007/s00268-010-0572-7)

26 Meijer WT, Grobbee DE, Hunink MG, Hofman A \& Hoes AW. Determinants of peripheral arterial disease in the elderly: the Rotterdam study. Archives of Internal Medicine 2000160 2934-2938. (https://doi.org/10.1001/archinte.160.19.2934)

27 Hooi JD, Kester AD, Stoffers HE, Overdijk MM, van Ree JW \& Knottnerus JA. Incidence of and risk factors for asymptomatic peripheral arterial occlusive disease: a longitudinal study. American Journal of Epidemiology 2001153 666-672. (https://doi.org/10.1093/ aje/153.7.666)

28 Wattanakit K, Folsom AR, Selvin E, Coresh J, Hirsch AT \& Weatherley BD. Kidney function and risk of peripheral arterial disease: results from the Atherosclerosis Risk in Communities (ARIC) Study. Journal of the American Society of Nephrology 2007 18 629-636. (https:// doi.org/10.1681/ASN.2005111204)

29 Matsushita K, Ballew SH, Coresh J, Arima H, Arnlov J, Cirillo M, Ebert N, Hiramoto JS, Kimm H, Shlipak MG, et al. Measures of chronic kidney disease and risk of incident peripheral artery disease: a collaborative meta-analysis of individual participant data. Lancet 2017 5 718-728. (https://doi.org/10.1016/S2213-8587(17)30183-3)

30 Selvin E, Kottgen A \& Coresh J. Kidney function estimated from serum creatinine and cystatin $\mathrm{C}$ and peripheral arterial disease in NHANES 1999-2002. European Heart Journal 200930 1918-1925. (https://doi. org/10.1093/eurheartj/ehp195)

31 D'Agostino RB, Sr, Vasan RS, Pencina MJ, Wolf PA, Cobain M, Massaro JM \& Kannel WB. General cardiovascular risk profile for use in primary care: the Framingham Heart Study. Circulation 2008117 743-753. (https://doi.org/10.1161/CIRCULATIONAHA.107.699579)

32 Echouffo-Tcheugui JB \& Kengne AP. Comparative performance of diabetes-specific and general population-based cardiovascular risk assessment models in people with diabetes mellitus. Diabetes and Metabolism 201339 389-396. (https://doi.org/10.1016/j. diabet.2013.07.002)

33 Chamnan P, Simmons RK, Sharp SJ, Griffin SJ \& Wareham NJ. Cardiovascular risk assessment scores for people with diabetes: a systematic review. Diabetologia 200952 2001-2014. (https://doi. org/10.1007/s00125-009-1454-0)

34 Zhao X, Zhang X, Ran X, Xu Z \& Ji L. Simple-to-use nomogram for evaluating the incident risk of moderate-to-severe lead in adults with type 2 diabetes: a cross-sectional study in a Chinese population. Scientific Reports 202010 3182. (https://doi.org/10.1038/s41598-019-55101-1)

35 Yang W, Zhao W, Xiao J, Li R, Zhang P, Kissimova-Skarbek K, Schneider E, Jia W, Ji L, Guo X, et al. Medical care and payment for diabetes in China: enormous threat and great opportunity. PLoS ONE 20127 e39513. (https://doi.org/10.1371/journal.pone.0039513)

36 Jiao XM, Zhang XG, Xu XU, Yi C, Bin C, Cheng QP, Gong QQ \& Lv XF Blood glucose fluctuation aggravates lower extremity vascular disease in type 2 diabetes. European Review for Medical and Pharmacological Sciences 201418 2025-2030.

37 Mohammedi K, Woodward M, Hirakawa Y, Zoungas S, Williams B, Lisheng L, Rodgers A, Mancia G, Neal B, Harrap S, et al. Microvascular and macrovascular disease and risk for major peripheral arterial disease in patients with type 2 diabetes. Diabetes Care 201639 1796-1803. (https://doi.org/10.2337/dc16-0588)

Received in final form 12 August 2021

Accepted 20 August 2021

Accepted Manuscript published online 23 August 2021 https://ec.bioscientifica.com https://doi.org/10.1530/EC-21-0152 (c) 2021 The authors Published by Bioscientifica Ltd

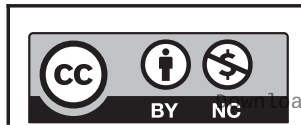

This work is licensed under a Creative Commons Attribution-NonCommercial 4.0 International License. ded from Bioscientifica com at 04/26/2023 12:16:10PM 